\title{
EL TECHO DE CRISTAL EN LA INTERMEDIACIÓN TURÍSTICA: EL CASO DE GALICIA
}

\section{THE GLASS ROOF IN THE TOURISM INTERMEDIATION SECTOR: THE CASE OF GALICIA}

1. Máster en Turismo, Universidade da Coruña. A Coruña, (España). E-mail: raquelsromo@gmail.com

2. Investigadora, Universidade da Coruña. A Coruña, (España). E-mail: larissa.gabriel@udc.es

3. Profesor e Investigador Escola de Turismo, Universidade da Coruña. A Coruña, (España).E-mail: jakson.soares@udc.gal

\section{Citación sugerida:}

Santiago Romo, R., Macêdo Castro Gabriel, L.P., y Rodrigues Soares, J.R. (2017). El techo de cristal en la intermediación turística: el caso de Galicia. 3C Empresa: investigación y pensamiento crítico, 6(3), $1-15$. DOI: <http://dx.doi.org/10.17993/3cemp.2017.060331.1-15/>. 


\section{RESUMEN}

La industria turística gallega presenta una plantilla primordialmente femenina, sin embargo, el techo de cristal se detecta en algunas empresas de intermediación del sector bastante jerarquizadas. Así, este estudio se concentra en analizar los discursos de trabajadoras de las empresas de intermediación gallegas con el objetivo de interpretar los valores, significados, estereotipos y sanciones que median las relaciones trabajo por género en el sector. Con este estudio esperamos ofrecer visibilidad a las barreras que provocan desperdicio de capital humano capacitado.

\section{ABSTRACT}

The Galician tourist industry has a predominantly female staff, however, the glass roof is present in some companies of the intermediation sector which are quite hierarchical. Thereby, this study concentrates on analyzing the discourses of female workers from the Galician intermediation companies with the aim of interpreting the values, meanings, stereotypes and sanctions existing in the relationship between work versus gender in the sector. With this study we hope to provide visibility to the barriers that cause waste of trained human capital.

\section{PALABRAS CLAVE}

Techo de cristal, empresas de intermediación, turismo, sistema de género, eficiencia empresarial.

\section{KEY WORDS}

Glass roof, intermediation companies, tourism, gender system, business efficiency. 


\section{INTRODUCCIÓN}

Las empresas de intermediación gallegas cuentan con una gran plantilla de mujeres entre su personal. Es sabido que las mujeres se forman más que los hombres en carreras de turismo, y específicamente en carreras más técnicas como son las especialistas en intermediación turística. Pero, el techo de cristal es un problema que se presenta en todas las industrias, y como no, también en la turística, donde se encuentran profesiones primordialmente femeninas.

Con todo, existen unas empresas bastante jerarquizadas donde, aunque la mujer tiene una considerable presencia, son los hombres los que sustentan los cargos de mando o dirección. Así, esta investigación viene a estudiar el sistema de género, es decir, los valores, normas, significados, roles, estereotipos y sanciones que median las relaciones trabajo por género en el sector de la intermediación turística. Para eso, se realizó un estudio de caso en las empresas de intermediación gallegas, concretamente de la provincia de A Coruña.

La metodología utilizada ha sido las entrevistas en profundidad a mujeres que trabajan en agencias de viajes y el análisis del discurso de las mismas. Como principales resultados, hemos encontrado que, aunque la sociedad evoluciona y esa situación parece para salir del "natural" y dirigirse a algo pasado, la primera función de la mujer es ser madre y cuidar de la familia, por eso se les relega a los puestos de menor impacto para la empresa. Con ese estudio esperamos ofrecer nuevos puntos de vista y soluciones a la pérdida de capital humano y consecuente merma de eficiencia debido a prejuicios cuanto a género.

El artículo está estructurado en $\mathrm{X}$ secciones, además de esta primera de carácter introductorio.

La segunda sección trata de la problemática del techo de cristal. En la tercera se conciben los procedimientos metodológicos. En la cuarta sección se realiza la presentación y discusión de los resultados, en cuanto que en la última sección se presentan las consideraciones finales del estudio.

\section{EL TECHO DE CRISTAL}

Si realizamos una breve recapitulación de las últimas décadas del mercado laboral desde una perspectiva de género, debemos señalar los años 80 como punto de partida de la reciente inclusión de la mujer en el mismo. En líneas generales y en lo que reguarda al ámbito nacional, se trata de un periodo en el que se produce un aumento paulatino de la presencia de las mismas y una mayor permanencia en los puestos de trabajo que anteriormente eran abandonados en detrimento del soporte de cargas familiares. Los factores que impulsan este cambio vienen dados, principalmente, por el movimiento de la liberación de la mujer acompañado del crecimiento económico que se produjo durante ese 
periodo y una serie de mejoras en el salario de ambos géneros, obteniendo como resultado un incremento de dotación de tiempo al trabajo remunerado (Cebrián López y Moreno Raymundo, 2008). De la mano de estos sucesos que sustentaron el avance de la mujer a nivel laboral, camina su acceso a la formación reglada en todas sus vertientes.

Adentrándonos en una temática específica, queremos señalar la relación titulación o formación con competitividad de las empresas. Girando la vista hacia el perfil curricular que obtienen los egresados en turismo se detectan como capacidades adquiridas el management, la gestión de recursos técnicos y humanos, la planificación y gestión de equipos, conocimientos de una o varias lenguas extranjeras, habilidades comerciales, etc. (García Isa, Fernández Gómez y Moreno Jorge, 2013) entre otras. . De este modo, se puede subrayar que se corrobora la relación entre conocimiento y competitividad. Con todo ello y con el turismo reconocido oficialmente como disciplina universitaria en la cual existe una mayor proporción de mujeres (Soares, Santiago Romo y Gabriel, 2016) podemos concluir que en el sector turístico se origina una "perfecta capacitación de la mujer para el desempeño de las actividades recomendadas en los distintos campos ocupacionales [...]" (García Isa, Fernández Gómez y Moreno Jorge, 2013).

Dados los factores anteriormente citados, poniendo el foco de atención en la intermediación del sector, se observa que la proporción de mujeres tituladas en turismo rebasa significativamente el número de hombres con la misma capacitación formativa, no obstante se produce una inversión en los datos en cuanto a la representación de ambos géneros si ponemos el punto de atención en la realidad laboral de este sector (Soares, Santiago Romo y Gabriel, 2016) y en concreto en el de la intermediación dentro del mismo. Desde este prisma, teniendo en cuenta que la eficiencia o la permanencia en el mercado de una empresa dependen directamente del "core-competence" así como de activos intangibles derivados del Know-how, surge una necesidad latente de conocer ¿por qué los puestos que van a determinar el posicionamiento de las empresas turísticas en el mercado no son llevados a cabo por la mujer? (dado que en mayor proporción, a priori, presentan una competencia profesional superior) ¿Por qué en el sector de la intermediación turística se desperdicia capital humano? ¿Cuáles componen las barreras que impiden que la mujer acceda a puestos para los que se halla perfectamente capacitada? Asimismo, teniendo en cuenta que se presentan elementos desincentivadores a nivel global en el terreno laboral desde la perspectiva de género tales como la precariedad laboral, la brecha salarial, la carga de trabajo doméstico unilateral (Toribio Muñoz, 2001) queremos conocer en qué medida influyen en el desempeño de puestos de management en el sector de la intermediación turística. Siguiendo este mismo discurso, se pretende abrir otra línea de investigación que permita conocer de qué modo todo lo mencionado, influye en la decisión de la mujer a la hora de elegir ocupar o no los puestos de responsabilidad en la intermediación turística para los que se hallan, como se cita anteriormente, perfectamente capacitadas.

Aludiendo a las cuestiones que previamente se han mencionado, si realizamos una síntesis podemos confirmar que el punto principal recae en indagar para lograr concretar lo que se denomina como Techo de Cristal en las agencias de viajes, es decir, exponer los obstáculos que frenan el empoderamiento de la mujer en este sector. A colación de lo anteriormente expuesto, resulta necesario aclarar que el objeto de estudio no se centra en desarrollar la 
definición en sí del término, si no en retratar la situación actual desde la perspectiva de género. En la búsqueda de respuestas al no acceso de la mujer a las altas esferas de la dirección, tanto en el sector turístico como en otras esferas, probablemente de manera automática la respuesta se halla en el "machismo", presentándose este término como un elemento abstracto que impide reconocer donde se encuentra específicamente el epicentro de la problemática que en el estudio se revela, con lo cual impera la exigencia de concretar, es decir, exponer en qué consiste de manera determinada y si existe la discriminación por razón de sexo y en el caso de que así sea traducir en parámetros de que se compone (lenguaje sexista, legislación, brecha salarial, educación, segregación horizontal y vertical, etc.) Se busca de este modo dar visibilidad a esta problemática dado que no se podrán ejecutar las medidas precisas para erradicarla si se desconoce qué o cuáles limitaciones resultan necesarias derribar. No obstante, a través del análisis se pretenden abrir otras hipótesis encaminadas a detectar barreras, que se proyectan como imprescindibles para conocer y cambiar la discriminación por razón de género en la promoción laboral, como el techo de cemento, el techo de diamante y el suelo pegajoso (Huete, Brotons y Sigüenza, 2016).

Por otra parte y poniendo el énfasis en la relevancia de la realización de un estudio que abarque esta temática, se considera que su valor viene dado por tres factores. El primero se corresponde con la eficiencia en el sector turístico, el Turismo engrosa el P.I.B. nacional, suponiendo un $11,1 \%$ del total (INE, 2015) y ocupa un posicionamiento de liderazgo a nivel internacional (factor económico) Si en la intermediación turística se cuenta con un capital humano específico necesitamos conocer porque se está desaprovechando(competitividad en el sector).Por último, "la actividad económica de la mujer desde una perspectiva de género y sus características diferenciadoras han permanecido al margen de las investigaciones [...]" (Toribio Muñoz, 2001: 40). Esto último supone la visualización de la problemática y el avistamiento de una luz hacia el camino tanto de la igualdad (factor social) como de la consecución de objetivos para un mejor desarrollo del sector.

\section{METODOLOGÍA}

La investigación se elaboró a partir de los discursos recogidos por medio de entrevistas en profundidad a trabajadoras que ocupan puestos directivos en empresas del sector turístico gallego. Fueron realizadas 7 entrevistas a trabajadoras del sector de intermediación turística en la provincia de A Coruña.

Se utilizó esta técnica porque con ella se busca la intensidad de las respuestas, no una cuantificación o representación estadística. La entrevista consiste en la colecta de información a través de una lista de preguntas propuestas por el entrevistador que debe ser fundamentada en el conocimiento adquirido durante la revisión bibliográfica de la temática de investigación (Acevedo y López, 1988). Los discursos levantados servirán para deducir las motivaciones profundas y los contenidos más ocultos del individuo (Cavassa, 2007). Las entrevistadas, o también informantes (Bisquerra Alzina, 1989; Poupart, 2008) ofrecen a los investigadores sus percepciones y experiencias acerca del tema investigado. 
En la Tabla 1 se puede verificar la posición de las entrevistadas dentro de la empresa.

\begin{tabular}{ccc} 
Individuo & Posición en la empresa & Duración \\
\hline Ent.1 & Directiva & 36 minutos \\
\hline Ent.E2 & Mando intermedio & 31 minutos \\
\hline Ent.E3 & Mando intermedio & 33 minutos \\
\hline Ent.E4 & Mando intermedio & 34 minutos \\
\hline Ent.E5 & Mando intermedio & 41 minutos \\
\hline Ent.E6 & Mando intermedio & 31 minutos \\
\hline Ent.E7 & Directiva & 32 minutos \\
\hline
\end{tabular}

Tabla 1. Descripción de las entrevistas.

Fuente: elaboración propia.

Las participantes fueron contactadas anteriormente y solo las que se interesaron en participar de la investigación fueron citadas para ser entrevistadas. Todas las conversaciones fueron grabadas llegando a cerca de 240 minutos de material en total. Se buscó que las informantes estuviesen cómodas al compartir sus vivencias con los investigadores. Las entrevistas se realizaron en sus locales de trabajo y en distintos horarios del día. Con eso, a partir de ahora se presentan los análisis realizados.

Para analizar los discursos se utilizó el Análisis Sociológico de Discursos, técnica que se preocupa con las motivaciones e intereses de los informantes (Alonso, 1998), es decir, se fundamenta en la interpretación del contexto de los individuos (Alonso, 2002). Conde (2009) desarrolló un conjunto detallado de orientaciones para el uso del ASD. Pero el autor resalta que estas orientaciones no deben ser vistas como una fórmula, sino que los análisis dependerán de la creatividad y singularidad de cada estudio. Soares y Godoi (2017) plasmaron las principales etapas del análisis propuestas por Conde en la siguiente Figura 1.

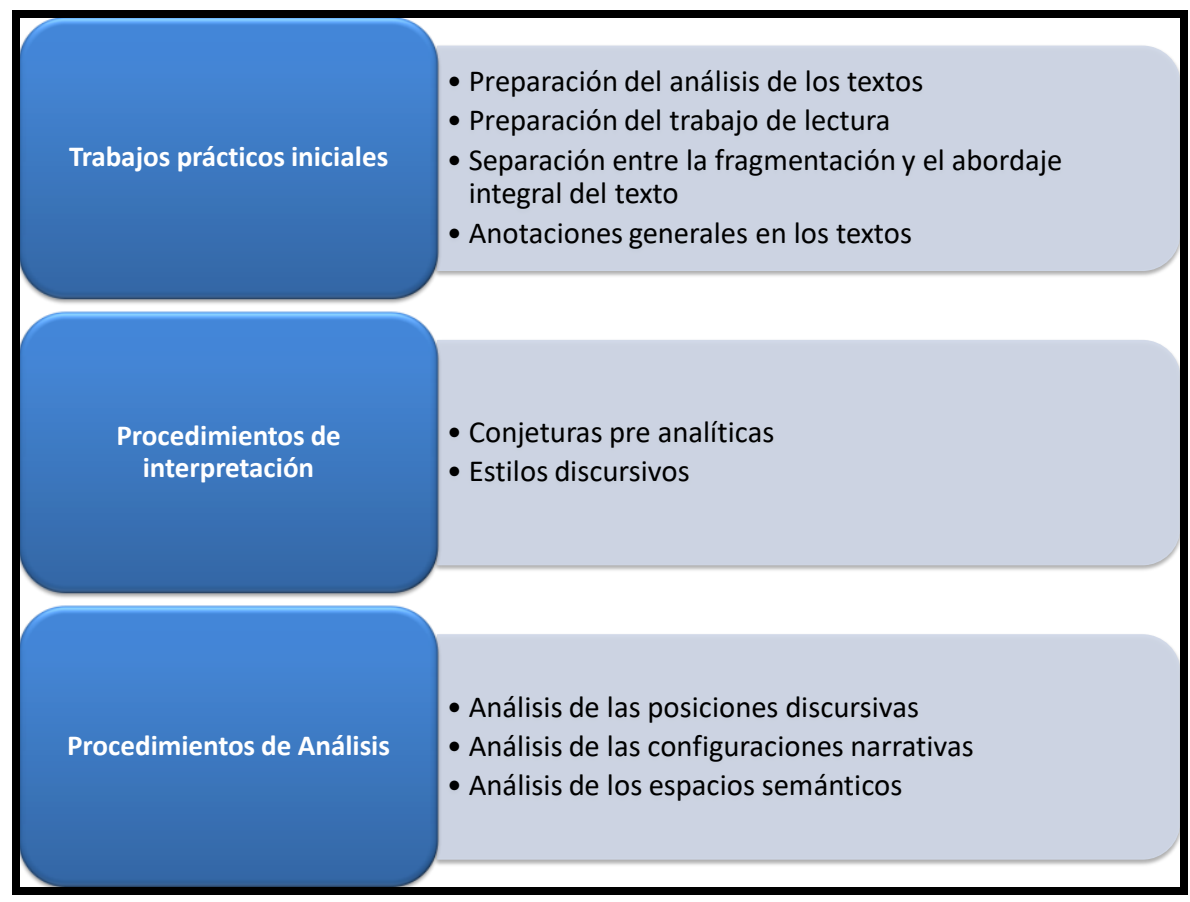

Figura 1. Procedimientos del Análisis Sociológico del Discurso

Fuente: Soares e Godoi (2017) adaptado de Conde (2010). 
Trabajos prácticos iniciales: en etapa se realiza la preparación y lectura sistemática del corpus, se anotan y clasifican sus contenidos, se busca contrastar y asociar sus significados de modo riguroso. Con esta etapa se estimula la creatividad del investigador con el surgimiento de los primeros insigths (o hipótesis).

Procedimientos de interpretación: en esta segunda etapa se realiza el diseño de las conjeturas pre analíticas que darán el sentido general del texto y el análisis de los estilos discursivos. Las primeras son consideradas como las intuiciones que dan sentido al objetivo de la investigación. Para hacer un buen diseño de las conjeturas es fundamental que quién investiga haga un análisis de la dinámica del corpus (texto resultante de las entrevistas). Para eso, haga uso de sus anotaciones e insights producidos durante la investigación. No solo eso, que busque leer y comprender las generalidades del texto indagando las relaciones con los objetivos del estudio (Conde, 2009). Ya los estilos discursivos representan las formas de expresión e singularidades de cada entrevistado. Se caracteriza por la identificación de conflictos ideológicos y simbólicos de los informantes delante de lo que les fue cuestionado.

En la llamada etapa de Procedimientos de Análisis es donde ocurre el ASD propiamente dicho. Se divide en: análisis de las posiciones discursivas, configuraciones narrativas y diseño de los espacios semánticos. Para realizar esta etapa, se busca responder las siguientes preguntas: ¿quién habla?, ¿desde qué posición se habla, se produce el discurso?, ¿qué es lo que está en juego en lo que se habla?, ¿qué se quiere decir con lo que se dice?, ¿de qué se habla?, y finalmente, ¿cómo se organiza e habla?

Se objetiva encontrar los puntos de vista sobre el tema de la investigación y la relación con las dimensiones ligadas al contexto histórico y social de los individuos, las posiciones sociales que expresan y lo que los sujetos abordan relacionados con los objetivos de la investigación (Conde, 2009). Para realizar el Análisis de las configuraciones narrativas, se debe considerar las tensiones, los conflictos, las opiniones y las diferencias de los posicionamientos de los interlocutores. Tiene como objetivo identificar para quién está dirigido el mensaje y qué se busca con el discurso (Coelho, 2012).

Finalmente, el Análisis de los espacios semánticos busca construir el conjunto de espacios semánticos expresados en el corpus que pueden ser importantes para llegar a los objetivos de la investigación. Consiste en la organización de las posibles asociaciones que los textos establecen entre sus elementos. Según Conde (2009) se trata de la organización de los contenidos y formas verbales en función de los objetos de la investigación.

Con todo y después de presentado los métodos de colecta y de análisis de los discursos, en el siguiente punto se presenta el Análisis Sociológico de los Discursos. Es decir, los resultados que se llegaron en formato de ASD. 
A partir de ahora se presentará la discusión de los resultados a partir de los discursos de las informantes.

\subsection{TRABAJOS PRÁCTICOS INICIALES}

En esta tapa se realizaron las transcripciones de las entrevistas incidiéndose con las anotaciones que ayudaron a responder los cuestionamientos de la investigación. Es decir, para producir las primeras intuiciones e insights, sensaciones, ideas generales y conclusiones acerca de lo investigado. Eso todo originó, posteriormente, las conjeturas pre analíticas. El material resultante de la transcripción fue leído desde la perspectiva del individuo, es decir, según las entrevistadas mismas abordaron determinados temas en sus discursos. Las anotaciones realizadas en la libreta de campo han servido para iluminar el contexto de las entrevistas.

\subsection{PROCEDIMIENTOS DE INTERPRETACIÓN}

El objetivo de este trabajo es identificar las dificultades encontradas por las mujeres en sus carreras laborales. Con todo, fueron entrevistadas trabajadoras del sector de la intermediación turística en la provincia de A Coruña. Hemos encontrado en los discursos que las dificultades de acceso a puestos directivos no son identificadas igualmente por unas y o otras trabajadoras. Es decir, dependiendo del puesto ocupado por la informante, ellas creen que hay más o menos dificultades. De esta manera, la primera conjetura de esta investigación es que cuanto más alto es el puesto del individuo en la empresa, menos crítico será su discurso con las dificultades encontradas para llegar hasta ahí.

Asimismo, aunque en este sector existe una presencia preponderante de mujeres, los perjuicios y el machismo rigen las impresiones a respecto del estilo de liderado de las mujeres. Con todo, se conjetura también que lenguaje y micro machismos son aspectos comportamentales que influyen en la posibilidad de la mujer acceder a los cargos directivos.

Como investigadores se busca identificar en el discurso los estilos de as hablas. Es decir, como se construye el discurso en la comunicación de sus informantes. En este sentido, hemos identificado en los discursos acerca del techo de cristal en la intermediación turística gallega que las informantes asumen posiciones relacionadas con los estilos de liderazgo y con el poder de decisión formal. No se quiere decir que siempre coincida con el liderazgo y poder de decisión real, es decir, hemos encontrado que aunque algunas decisiones son tomadas formalmente por ellas, pero ellos son los que repasan para el resto de la empresa. Por último, hemos de destacar que se identificaron algunos estilos discursivos en las entrevistas. Desde madres trabajadoras, trabajadoras con cargas familiares o lideresas, por ejemplo. Pero finalmente, se hace hincapié a la situación machista encontrada en las 
palabras de una de las entrevistas que no dice que "las mujeres directivas son mandonas y los hombres son líderes".

\subsection{PROCEDIMIENTOS DE ANÁLISIS}

El primer análisis es el de las posiciones discursivas. Sirve para guiar los siguientes análisis. Con ese análisis se busca responder las dos primeras preguntas: ¿quién habla?, y ¿desde qué posición se habla, se produce el discurso? En este sentido, podemos caracterizar los discursos de las entrevistas en tres bloques sociales: un primer grupo que afirma inequívocamente que la igualdad de oportunidades existe; otro que percibe que la igualdad no existe; $y$, el que afirma que en la actualidad la equidad es inexistente pero pronto va cambiar.

En el caso del primer grupo, con ese discurso las informantes explican y ejemplifican la igualdad de oportunidad es real en su industria. Este bloque está formado por los discursos ofertados por los individuos que ya están ocupando cargos de dirección (E1 y E7). Los discursos de las informantes que están empezando sus carreras, es decir, que han asumido cargos de mando intermedio, pueden ser encontrados en los otros dos grupos. Por un lado encontramos (E4, E5 y E6) que coinciden en el pesimismo de la situación y en que la sociedad no va cambiar para que las mujeres puedan acceder en la empresa turística de la misma manera que los hombres. Y por otro lado encontramos (E2 y E3), que asumen posiciones que afirman que en la actualidad la equidad es inexistente, pero que gracias a las nuevas generaciones preparadas para una sociedad más justa eso será posible.

De esta manera, deducimos que estas tres posiciones asumidas por las informantes que caracterizan estos tres grupos, si fueron identificadas en las entrevistas, muy probablemente también lo serán en la vida social de la industria turística.

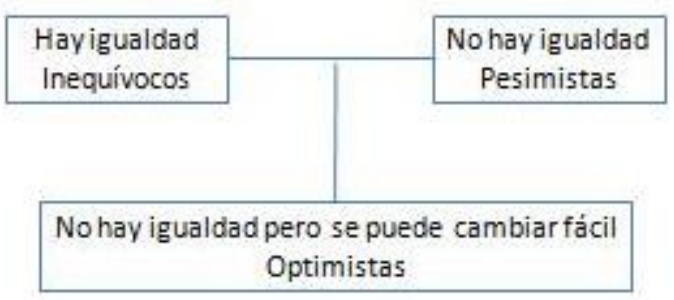

Figura 2. Mapa de posicionamiento de las entrevistas. Fuente: elaboración propia.

Para realizar el análisis de las configuraciones narrativas se debe responder las preguntas: ¿qué es lo que está en juego en lo que se habla? y ¿qué se quiere decir con lo que se dice? Esta etapa se encuentra representada por el Triángulo Sémico de Lévi-Strauss constituido a partir de tres vértices que representa el natural, el cultural y el artificial de una situación social. Según Conde (2009), un fenómeno social que hoy es visto como "artificial" en una sociedad, mañana puede estar más integrado y percibirse como "cultural", y luego más tarde, puede estar tan más integrado y domesticado que se inscriba en un plano casi imperceptible, impregnado en la conciencia del sujeto. Es decir, acabando por ser encarado como algo "natural". 


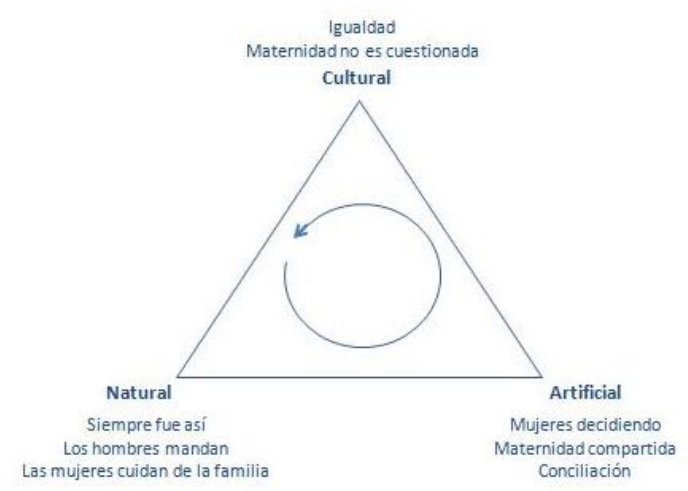

Figura 3. La maternidad desde lo artificial a lo natural en la empresa.

Fuente: elaboración propia.

Hemos identificado en los discursos de las informantes que es "natural" para la sociedad que las mujeres abran mano de sus carreras profesionales para dedicarse al cuidado de la familia. En la sociedad en la cual estamos realizando este trabajo (en el contexto gallego y en la industria turística), podríamos incluso llegar a afirmar que en cuanto la figura "maternidad" no entra en el tema de discusión, las informantes afirman que el capital humano es valorado por la empresa de la misma manera. Pero, cuando se pasa a cuestionar el techo de las empresas, la situación "natural" hoy es que esta figura de mando va a ser ejercida por un sujeto que no dedique, o no tenga carga familiar.

Mirando el triángulo propuesto, vemos que las empresas que tienen mujeres en puestos de liderazgo, con poder de decisión, son vistas como una situación artificial. Las informantes nos indican que la sociedad no está preparada para ofrecer conciliación laboral y familiar y, aunque haya mujeres en puestos de mando, es completamente artificial que ella no sea también quien lleve el peso del cuidado de su familia. Esta situación es artificial porque aún falta mucho por consolidar políticas (públicas o privadas/empresariales) de conciliación o maternidad compartida que impactarán directamente en el cambio social con relación a la igualdad en la empresa turística.

El último vértice, el "cultural", las informantes nos hacen conjeturar que este momento llegará cuando la maternidad no sea cargada de prejuicios en la consideración de las empresas. Es decir, puede que intencionadamente o no, las empresas, al igual que las personas, siempre consideran que la mujer tiene un papel principal en su vida, ser madre. Ya se han visto cambios en este sentido, disminución de la natalidad o aumento de la edad media de tener hijos, por ejemplo. Pero eso siempre es una "carga" que lleva la mujer, quién no se concibe la idea de que no nacieron solo para tener hijos.

Bien, la problemática que se quiere llegar es que no todas las mujeres quieren ser madres o no todas ellas pueden ser madres, por lo tanto, es injusto que se les implique ese papel como el principal rol para su realización personal. En el momento que eso sea entendido por la sociedad como una carga, es probable que la igualdad esté más cerca. En este momento, quiera sea por conciliación familia-trabajo, quiera sea porque hombres y mujeres compartan la mochila del cuidado del recién nacido (no de la maternidad), es cuando las empresas van entender que el capital humano aportado por sus trabajadores va independer 
de su género, y más bien, dependerá de su formación. Por ello, tanto ganarán empresas, como sociedad.

Por último, para realizar el análisis de los espacios semánticos se busca responder las preguntas: ¿de qué se habla? y ¿cómo se organiza e habla? Se puede afirmar que, aunque se esté trabajando en una industria que cuenta con una presencia masivamente femenina, los discursos y sus consecuencias para las tomas de decisiones dentro de las empresas están permeados de percepciones cargadas de machismo. En este sentido, los discursos de las informantes se construyeron a partir de atractores semánticos como los siguen: años de formación, trabajos sumisos, maternidad, jefes/jefes de familia, desapego familiar.

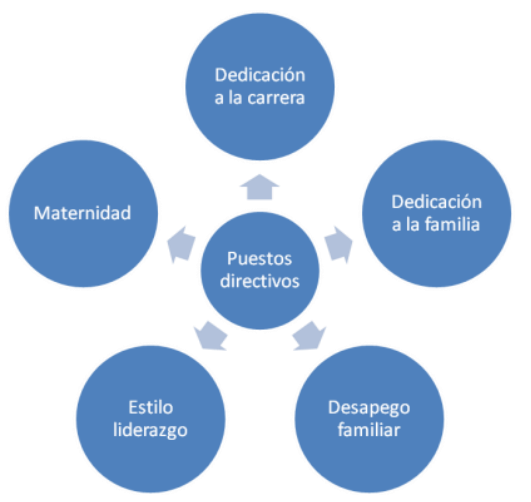

Figura 4. Espacios semánticos problemas para puestos directivos femeninos. Fuente: elaboración propia.

Hemos identificado que se espera que la mujer sea más eficiente que el hombre para que pueda tener sus mismas oportunidades. Por la contra, también hemos analizado que esta generación, que hoy toma decisiones, está siendo sustituida paulatinamente por individuos formados en valores y capacidades que ultrapasan los prejuicios. Si bien es cierto, los discursos se forman a partir de esos atractores semánticos, pero se conforman desde varias perspectivas. Una, optimistas en cuanto a la mejora en un corto espacio de tiempo; dos, pesimistas en cuanto a que, si la educación formal prepara el individuo hacia una sociedad más igualitaria, los medios de comunicación, el lenguaje o los micro machismos minimizarán la consecución de dicha igualdad.

Aunque se percibe que la formación prepara a las mujeres para la toma de decisión, para puestos de dirección, la sociedad sigue reticente, teniendo prejuicios en cuanto a la hora de dar un puesto como este a una mujer. Asimismo, eso no es una cuestión ni definitiva, ni tampoco sin solución. Ella ocurre debido a que la región donde se realizó el estudio aun cuenta con muchas empresas de calado familiar. Es decir, muchas veces administradas a la sombra por un empresario/inversor/director de sexo masculino. Con eso, cuando surge la necesidad de contratación de una persona capaz de tomar decisiones acertadas para la empresa, muchas veces los prejuicios superan el capital humano. 


\subsection{RESULTADOS}

A pesar de la existencia de consciencia de cierta paridad por parte de la mujer, según los datos del mercado y a partir de los discursos, la equidad de género se presenta como un factor incipiente en las empresas de intermediación turística. Asimismo, hemos detectado que cuanto más se aproximan a desarrollar un cargo en las altas esferas de dirección en el entorno empresarial, existe una mayor convicción de la existencia de igualdad, obviando de este modo las dificultades añadidas a la contratación de mujeres para ocupar cargos directivos.

La consecución de puestos directivos se halla fuertemente ligada a la elección personal entre el desarrollo de una carrera profesional y la conciliación con la vida familiar o personal para la mujer, lo que se traduce en una incesante lucha para equilibrar los compromisos profesionales y familiares. Es decir, para avanzar profesionalmente la mujer se ve obligada a elegir entre una u otra opción o en cambio, a decantarse por ambas y soportar una mayor carga de trabajo.

La sociedad impone las reglas, en cuanto a que desde la perspectiva de la misma y desde un enfoque global, se considera que la prioridad "natural" de la mujer recae en el desempeño de actividades no remuneradas estrechamente ligadas al entorno familiar relegando su actividad profesional a un segundo plano. Si ponemos el acento en este hecho, para las informantes, este pensamiento generalizado se produce porque culturalmente se asocia el rol de la mujer con los cuidados, es decir, el imaginario social esboza el perfil de la mujer cómo el de aquélla mejor preparada de manera natural para encargarse de tareas relacionadas con el cuidado de sus semejantes.

En cuanto a las diferencias de liderazgo entre ambos géneros, las entrevistadas no perciben distinciones en el modus operandi de hombres y mujeres. No obstante, exponen que las habilidades personales ligadas a la condición de líder tales como resolución, capacidad de negociación, asertividad, entre otras, presentan un matiz peyorativo cuando despuntan en la mujer. Es decir, mientras que estas características se asumen como válidas y positivas en el líder masculino, en cuanto a lo que respecta a la mujer, se transforman adquiriendo connotaciones negativas. De este modo, cuando la mujer demuestra las aptitudes anteriormente citadas, la óptica sufre una transformación convirtiéndolas en rasgos negativos ("mandona", "explosiva", etc.). Con lo cual, culturalmente las mismas habilidades que se valoran para un puesto de liderazgo se catalogan en diverso modo si lo desempeña una mujer o un hombre, descartando la capacitación y preparación de la mujer para ejercer las mismas funciones de liderazgo.

En relación al progreso de la mujer en el ámbito corporativo, ellas relacionan las dificultades de promoción con el resultado de las diferencias de estilo y la ausencia de valoración de un perfil alternativo por parte de los reclutadores y personal de Recursos Humanos.

Los términos "prejuicio" y "machismo" se suceden constantemente en el corpus del estudio, se detecta como una señal de una sociedad, en concreto de una industria turística, 
arraigada todavía a sentimientos peyorativos en lo que se refiere a la dirección de las empresas llevada a cabo por la mujer.

Si hacemos hincapié en la percepción social de los roles de la mujer con respecto al management, teniendo en cuenta que el acceso de la mujer a la Universidad y a la formación, en líneas generales, se descifra como una barrera abatida, se hace necesario proponer un cambio de paradigma para obtener una igualdad real de oportunidades en el sector de la intermediación turística.

\section{CONCLUSIONES}

Quizás lo primero y más importante a destacar de este trabajo es que, más que diferencias de estilo de liderazgo, se identificó que las mujeres no acceden a puestos directivos debido a aspectos más relacionado con la sociedad que con su formación. Es decir, el problema representa un desafío mucho más grande a resolver. La solución en cuanto a la igualdad de acceso a puestos directivos exige soluciones nada simples o fáciles de implementarse. Es decir, hace falta un cambio social desde lo más básico visto que aunque la formación es algo fácilmente solucionable (son años y años de acceso de la mujer a formación en todos los ámbitos sociales), eso aún no llegó a tener la repercusión en el mercado laboral.

Analizando el discurso de las entrevistadas en agencias de viajes, podemos afirmar que independientemente del color político, existe consenso a la hora de identificar la maternidad como una barrera para conseguir desarrollar una carrera profesional. Es decir, aclarando que se detecta que la problemática radica en cómo se plantea esta situación actualmente, dado que empuja a la mujer a elegir entre maternidad o carrera profesional.

Del mismo modo y aunque una de las participantes reparte las tareas equitativamente, los discursos coinciden en la falta de una legislación real en materia de conciliación familiar que evite la segregación horizontal. Asimismo, esta situación requiere de concienciación por parte de los hombres y una mayor implicación para compartir la carga doméstica. Lo cual nos encamina a conjeturar que el planteamiento legislativo en cuanto a permisos no resulta beneficioso para la mujer a nivel laboral, si no existe una ley igualitaria para hombres y mujeres.

Asimismo, aunque se observe que existe presencia femenina en los mandos intermedios, todavía no se ha avanzado a las altas esferas de la dirección. Esta condición en parte, y a través de los discursos, viene dada por factores machistas, es decir, si cuestionamos el porqué de este hecho, no se halla un razonamiento lógico. Pero si es cierto que ellas identifican que la maternidad les relega a determinados puestos simplemente porque es natural que las mujeres sean madres antes de ser profesionales.

Para finalizar, se observa que los hombres que ocupan puestos de dirección delegan responsabilidades en las mujeres que componen su equipo, lo que se traduce en mayor esfuerzo y carga de trabajo percibiendo un menor salario, una vez más impidiendo el empoderamiento de la mujer. 
Desde la perspectiva de la realización de futuros trabajos, resultaría positivo y recomendable la aplicación de esta técnica, en estudios de diversos sectores dentro de la industria turística. Otra línea que se presenta como especialmente atractiva, se concentra en el análisis comparativo de los discursos de hombres y mujeres con respecto a la temática expuesta, así como de cotejarlos en otros sectores turísticos. Además, se sugiere la práctica de otras metodologías en cuanto a la recogida de datos como por ejemplo los grupos de discusión.

En relación a las limitaciones, la principal, una vez que se ha definido el tema, se detecta en el proceso de recogida de material. Uno de los inconvenientes recae en el reducido número de entrevistas a trabajadoras, de un único sector y ubicadas en una determinada zona de la provincia de A Coruña, este hecho se contrarresta en cuanto que, el estudio, forma parte de un proyecto de investigación de mayor amplitud.

\section{REFERENCIAS BIBLIOGRÁFICAS}

Acevedo, A. I.; López M., A. F. (1988). El proceso de la entrevista: conceptos e modelos. México, D. F.: Limusa Noriega Editores.

Alonso, L. E. (1998). La mirada cualitativa en sociología. Madrid: Fundamentos.

Alonso, L. E. (2002). "Los mercados lingüísticos o el muy particular análisis sociológico de los discursos de Pierre Bourdieu". Revista de Estudios de Sociolingüística, 3(1):111-132.

Bisquerra Alzina, R. (1989). Métodos de investigación educativa. Guía práctica. Barcelona: Ediciones Ceac.

Cavassa, C. R. (2007). Marketing Turístico. Análisis motivacionales. Comercialización y política de distribución. Ingeniería de la imagen, comunicación comercial y publicidad turística. México D. F.: Trillas.

Cebrián López, I.; Moreno Raymundo, G. (2008). La situación de las mujeres en el mercado de trabajo español. Desajustes y retos. Revista Economía industrial, 367, 121-137.

Coelho, A. L. (2012). Construção do discurso da sustentabilidade: uma prática de Análise Sociológica do Discurso no campo organizacional. Tese (Doutorado em administração e turismo). Universidade do Vale do Itajaí, Biguaçu.

Conde, F. C. (2009) Análisis sociológico del sistema de discursos. Cuadernos Metodológicos 43. Madrid: Centro de Investigaciones Sociológicas (CIS).

Conde, F. C. (2010) Análisis sociológico del sistema de discursos. Madrid: Centro de Investigaciones Sociológicas.

Instituto Nacional de Estadística (2015). Cuenta Satélite del Turismo de España. 
García Isa, I., Fernández Gómez, M., Moreno Jorge, M. (2013). El perfil ocupacional de los titulados en turismo dentro del sector turístico. Gran tour: revista de investigaciones turísticas, 7, 97-117.

Huete, R., Brotons, M. y Sigüenza, M.C., (2016). La desigualdad entre mujeres y hombres en el sector hostelero español. Estudios y Perspectivas en Turismo, 25, 73-87.

Poupart, J. (2008). A entrevista de tipo qualitativo: considerações epistemológicas, teóricas e metodológicas. In: A Pesquisa qualitativa: enfoques epistemológicos e metodológicos. Petrópolis: Vozes, 215-253.

Soares, J. R. S., Santiago Romo, R. y Gabriel, L. P. M. C. (2016) Empoderamento da muller na xestión do turismo: Estudo sobre a igualdade nas empresas turísticas de Galicia. In: Libro de Actas I Congreso Iberoamericano de Turismo y Responsabilidad Social, 90.

Soares, J. R. S.; Godoi, C. K. (2017). A metodologia da análise sociológica do discurso em estudos turísticos: o processo de transformação da imagem turística e sua relação com a lealdade. PASOS, Revista de Turismo y Patrimonio Cultural 15(1), 245-260.

Toribio Muñoz, M. R. (2001). La actividad empresarial femenina: ¿una superación de las trabas en el mercado laboral? Boletín Económico de ICE. $\mathrm{n}^{\circ} 2690$ del 30 de abril al 13 de mayo de 2001, 39-52. 